\title{
Mechanoreceptors of the Achilles tendon: a histomorphological study in pigs with clinical significance for humans
}

\author{
Stylianos Kapetanakis ${ }^{1}$ \\ Grigorios Gkasdaris ${ }^{1}$ \\ Eleni Daneva² \\ Panagiotis Givissis ${ }^{3}$ \\ Jannis Papathanasiou ${ }^{1}$ \\ Theodoros Xanthos ${ }^{4}$
}

1 Spine Department, Interbalkan European Medical Center, Thessaloniki, Greece

2 National and Kapodistrian University of Athens, Medical School, Athens, Greece

3 First Orthopaedic Department of Aristotle University of Thessaloniki, Papanikolaou Hospital, Exohi, Thessaloniki, Greece

4 Hellenic Society of Cardiopulmonary Resuscitation, Athens, Greece; European University Cyprus, School of Medicine, Nicosia, Cyprus

Corresponding author:

Stylianos Kapetanakis

Spine Department,

Interbalkan European Medical Center

Asclepiou 10

57001 Thessaloniki, Greece

E-mail: stkapetanakis@yahoo.gr

\section{Summary}

Introduction: Tendons contain neurosensory elements called mechanoreceptors which contribute to the neuromuscular system as sources of reflex signals. The literature is lacking in histological assessment of mechanoreceptors of the Achilles tendon in piglets and our aim was to indicate their types, location and quantity.

Methods: The study was performed using histological tissue samples from the Achilles tendon of ten healthy pigs, five left, five right, six males, four females. The samples were taken up to 12 hours after death. Immediately after removal, the tendons were placed in the laboratory where sections were taken and examined microscopically. The tendons were stained with the gold chloride method.

Results: The results showed that Golgi tendon organs, free nerve endings and Pacinian-like corpuscles were found in the Achilles tendon of pigs. Most structures were near the osteotendinous and myotendinous junctions, away from the middle portion of the tendon.

Conclusion: As shown in other studies and similarly in ours, mechanoreceptors tend to be close to the distant thirds and not in the middle third of the tendon. This study could have clinical application on human Achilles tendon and its repair after damage.

Level of evidence: IV.

KEY WORDS: Achilles tendon, gold chloride, Golgi, mechanoreceptors, Pacinian, pigs.

\section{Introduction}

Ligaments are considered to be passive structures that support and stabilize the movement. In addition, they tend to limit excessive movement of joints. Tendons serve to attach muscles to bones. In 1860, Krause described for the first time the presence of mechanoreceptors in ligaments ${ }^{1}$. Mechanoreceptors appear to transmit information to the brain about the position and the movement of muscles and bones in space. There are four basic types of mechanoreceptors which have been described: 1) Ruffini's corpuscles or endings type I; 2) Pacinian corpuscles or endings type II; 3) Golgi tendon organs or endings type III; 4) free nerve endings ${ }^{2,3}$. Many variations of mechanoreceptors have been described in several ligaments of the body. The most frequent ligament studied in humans and animals is the anterior cruciate ligament of the knee because of its importance in maintaining the stability of the knee ${ }^{3,4}$. A tendon of similar importance for the proper movement of the limbs is the Achilles tendon, which has many functions and has not been described extensively in the past as far as morphological aspects are concerned. Ruffini's type I corpuscles are stationary and occur primarily on the surface of the joints. Pacinian corpuscles are mostly described in common capsules, not in ligaments, and they are triggered by acceleration and fast motion of the joint. The more lamellae that are present, the more sensitive they are to the phasic component of the stimulus, culminating in the exquisite vibration sensitivity of the Pacinian corpus$\mathrm{cle}^{5}$. Golgi tendon organs or type III endings are located mainly in myotendinous and in osteotendinous junction, and their function is to receive signals about the direction of motion and the position of the joint in space $^{6}$. As regards to muscle spindles, they convey 
length information to the central nervous system via sensory neurons. This information can be processed by the brain to determine the position of body parts ${ }^{7}$. Finally, free nerve endings are pain receptors and it is doubtful whether they should be regarded as mechanoreceptors ${ }^{4}$.

The Achilles tendon is considered the thickest and strongest tendon of the body. It is located at the rear lower portion of the leg serving to attach the gastrocnemius, soleus and plantaris muscle to the calcaneous bone (Fig. 1). Similar to other tendons, the Achilles tendon contains different types of mechanoreceptors that support the role of the tendon in order to stabilize the movement of the body. There are few reports on the innervation of the Achilles tendon and the presence of mechanoreceptors ${ }^{8-10}$.

In this study, we examined the Achilles tendons of 10 pigs and the samples were processed using the gold chloride staining method in order to visualize and distinguish all structures. The purpose of this study was to indicate all types of mechanoreceptors, their location and their number. As far as we know, there has not been any similar morphologic study of the Achilles tendon concerning pigs yet.

\section{Materials and methods}

The experimental protocol was approved by the General Directorate of Veterinary Services of the Prefecture of Attica according to the Greek legislation on ethical issues and experimental procedures. Ethics in our study was based on the protection of animals used for experimental and scientific purposes and promotion of science with fair means, using adequate measures to minimize pain or discomfort. The study meets the ethical standards of the journal as stated in the article of Padulo et al. $2016^{11}$. The selection of these piglets depends on the correspondence of the anatomical and physiological characteristics of the animal with those observed in humans. All of them came from the same farm (Validakis, Athens). The histological study was performed on tissue samples from the Achilles tendons of 10 healthy piglets, breed Landrace Large White, weighing about $19 \pm 2 \mathrm{~kg}, 10-$ 15 weeks old, five left, five right, six males and four females without degenerative or rheumatic disease and they were taken up to 12 hours after death. Samples were retrieved aseptically from the hind limbs at the research facilities of the Experimental-Research Center in Pikermi, in Attica. Immediately after removal, the tendons were placed in normal saline and transported to the laboratory where sections were immediately cut $0.5 \mathrm{~mm}$ thick and examined under an optical microscope. They were stained with the gold chloride staining method, as modified ${ }^{12}$. Similar studies have also used this staining method $^{13,14}$.

The tendons were divided into thirds. The proximal $1 / 3$ was close to the myotendinous junction, the distant $1 / 3$ close to the osteotendinous junction and the rest the middle part of the tendon. A few millimeters of muscle and bone were removed with the insertion of the ligament to ensure a true muscle-tendon and bone-tendon specimen. Longitudinal and transverse sections were taken per tendon and the next steps followed. Initially, each tendon was placed in a sepa-

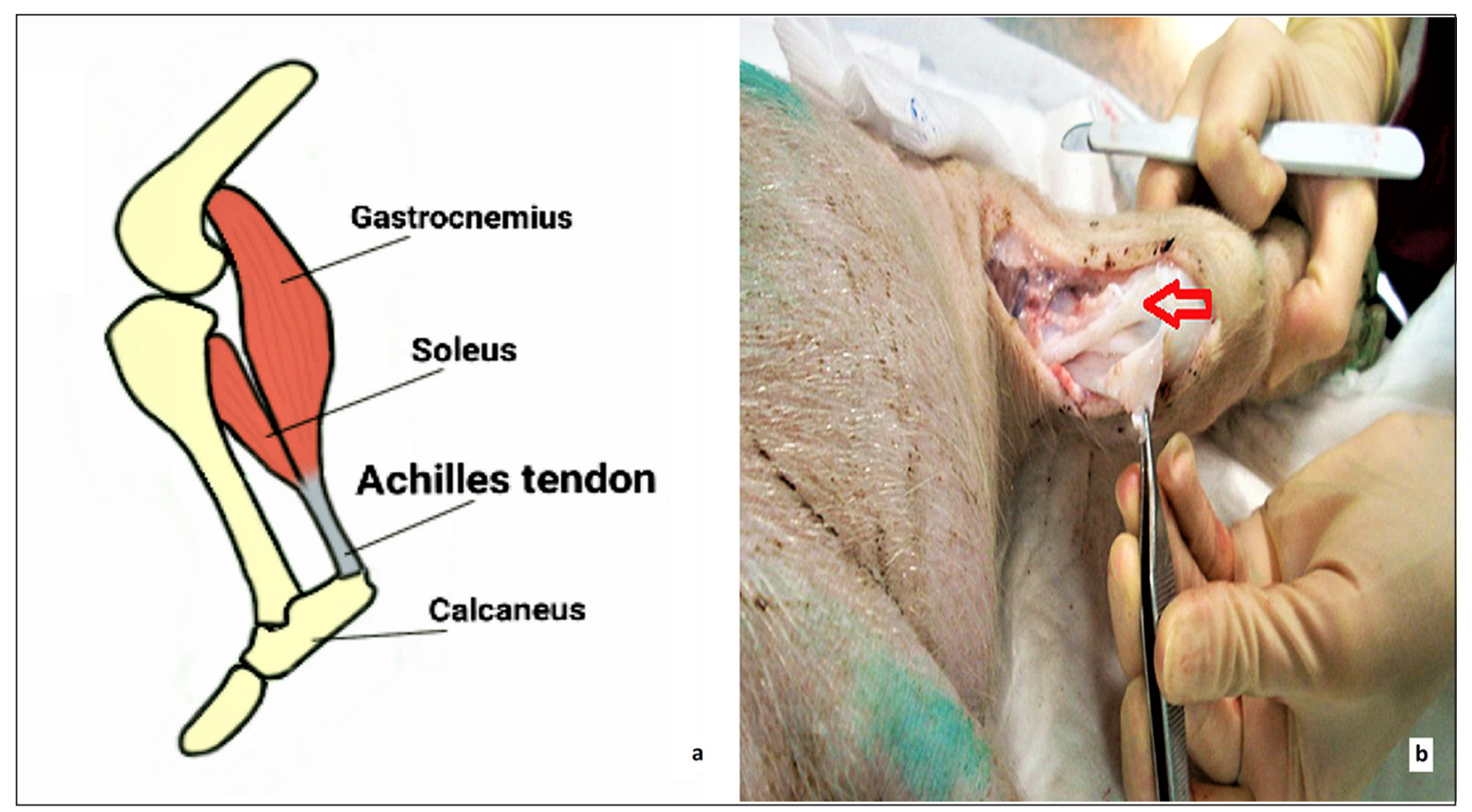

Figure 1. The Achilles tendon of a Landrace Large White pig (a) depicted on a schematic illustration and (b) photographed during the dissection process (as indicated with the red arrow). 
Table I. Descriptive statistics of Golgi marker.

\begin{tabular}{lllll}
\hline Mean value (MV) & Standard Deviation (SD) & Median & Interquartile range (IQR) & Min-Max \\
\hline 10,10 & 1,59 & 10,50 & 2,25 & $7-12$ \\
& Shapiro-Wilk & Statistic & df & Sig. \\
& & 0,912 & 10 & 0,295 \\
\hline
\end{tabular}

Table II. Descriptive statistics of Pacini marker.

\begin{tabular}{lllll}
\hline Mean value (MV) & Standard Deviation (SD) & Median & Interquartile range (IQR) & Min-Max \\
\hline 2,4 & 1,9 & 2,5 & 4,25 & $0-5$ \\
& Shapiro-Wilk & Statistic & df & Sig. \\
& & 0,890 & 10 & 0,170 \\
\hline
\end{tabular}

rate flask, covered by a solution of lemon juice and formic acid $88 \%$ in a ratio of $3: 1$, respectively, and was left in the dark for 10 minutes. Subsequently, the initial solution was replaced by a solution of $1 \%$ gold chloride and each flask was placed in dark place for 10 minutes. Then the second solution was substituted with formic acid solution $25 \%$ and placed in the dark for 30 minutes. The next step involved 3 washes of ethanol $70 \%$ for 10 minutes each. Afterwards, each sample remained in glycerol for 24 hours, from where, the samples were enclosed in paraffin blocks and then sections were obtained by microtome $(100 \mu \mathrm{m})$, followed by examination under the microscope.

The statistical analysis of this study was performed with the statistical package SPSS, version 16.00 (SPSS Inc, Chicago, IL). All tests are two-sided. The $p$-value $<0.05$ was determined as statistically significant difference level. Also the marginally statistically significant differences are recorded $(0.05<\mathrm{P}<0.1)$. Values of continuous variables are presented using the number of participants $(\mathrm{N})$, the mean value (MV), the standard deviation (SD), the median and the interquartile range (IQR). The frequency and the perspective percentages are used for the categorical variables. The control for the regularity of the distribution of the measurements was made using the Shapiro-Wilk test (due to the small number of observations $n=10$ ) and the normal probability plot. The correlation between the variables was studied with the Pearson and Spearman correlation coefficient.

\section{Results}

Only three types of mechanoreceptors were found: free nerve endings, Golgi tendon organs and Pacinian-like corpuscles. The Golgi variable is normally distributed under Shapiro-Wilk test. The mean value of the receptors was 10.1 with a standard deviation of 1.6. Minimum was 7 and maximum was 12 (Tab. I). The Pacini variable is normally distributed according to the Shapiro-Wilk test. The mean value of the receptors was 2.4 with a standard deviation of 1.9 . The minimum value in our sample was 0 and maximum was 5 (Tab. II). We observe that the free nerve endings were abundant in all the experimental animals. As regards to the correlation between the Golgi and the Pacini markers, there is a positive $(r=0.398)$ but not statistically important correlation, probably due to the small number of samples (Tab. II, Fig. 2). A brief look on the overall data is expressed on Figures 2, 3 and Table III.

The Pacinian-like corpuscles appeared to be ovalshaped with a fusiform centre and a few surrounding lamellae (Fig. 4a). The Golgi tendon organs were found aligned with the long axis of the tendon within the collagen bundles. An axon is seen entering and the structure exhibits a spiralled appearance and a spindle shape (Fig. 4b). Free nerve endings were observed as thin distinct strands (Fig. 4c).

We observed that Pacinian-like corpuscles and especially Golgi tendon organs were mostly found close to osteotendinous and myotendinous junctions and not in the middle third of the tendon. Cross sections and longitudinal sections were taken and different numbers of these three mechanoreceptors were found. To illustrate the variability of our findings we should refer to the complete lack of any mechanoreceptor other than free nerve endings in a section taken from the middle part of the Achilles tendon.

\section{Discussion}

Mechanoreceptors are corpuscles that have been identified in all ligaments and tendons of humans and animals. Their main function is to receive signals and transmit information to the brain, to improve the coordination of movement and prevent any injuries ${ }^{4}$. They tend to appear nearby the osteotendinous junction ${ }^{15}$. From 1860, when Krause ${ }^{1}$ described for the first time the presence of these structures, there has been extensive research on the proprioception of the neuro- 

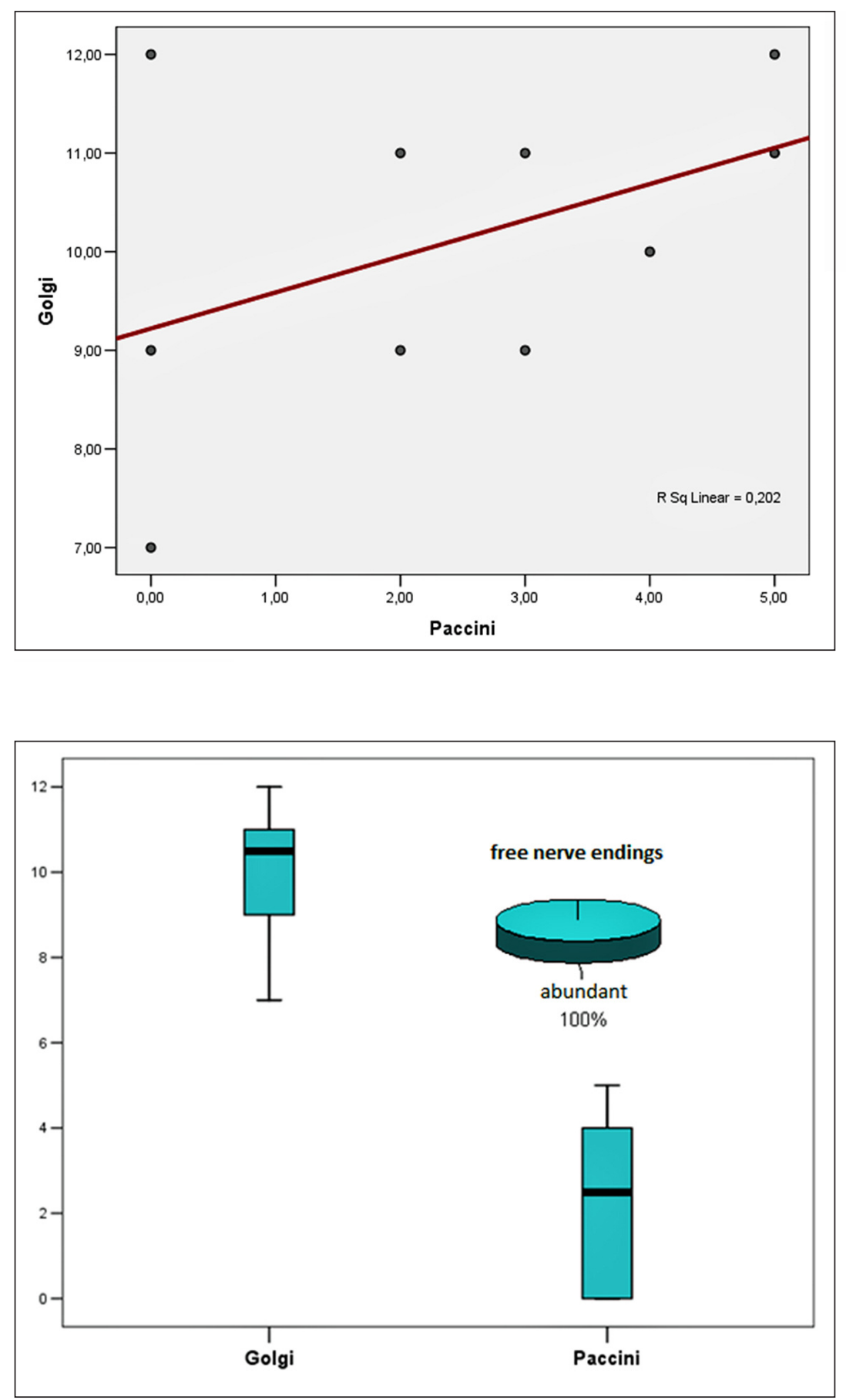

Figure 2. Correlation between the Golgi tendon organs and the Pacinian corpuscles.

Figure 3. Summary of the data of mechanoreceptors found on each pig.

Table III. Correlation of Golgi and Pacini markers.

\begin{tabular}{lll}
\hline & & Pacini \\
\hline \multirow{3}{*}{ Golgi } & Sperman & 0,398 \\
& p-value & 0,255 \\
& Pearson Correlation & 0,449 \\
& p-value & 0,193 \\
\hline
\end{tabular}



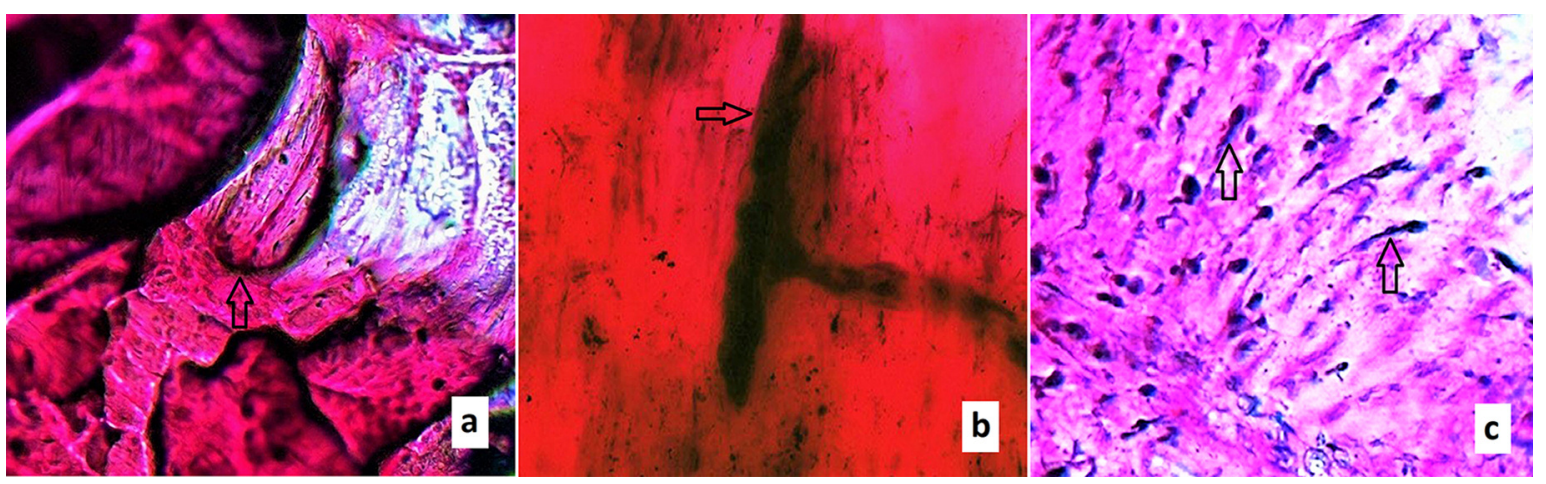

Figure 4 a-c. Longitudinal sections with the use of the gold chloride staining method. a. A Pacinian-like corpuscle is depicted (arrow). The Pacinian corpuscles appear to be oval-shaped with a fusiform centre and a few surrounding lamellae; $b$. a Golgi tendon organ is depicted (arrow). The Golgi tendon organs are found aligned with the long axis of the ligament within the collagen bundles. An axon is seen entering and the structure exhibits a spiralled appearance and a spindle shape; c. abundant free nerve endings (arrows) are indicated. Free nerve endings are observed as thin distinct strands.

muscular system and the role of mechanoreceptors on different tendons, ligaments, joints ${ }^{16-18}$.

In our study, we proceeded into slices of the tendons and we processed the samples using the gold chloride staining method, the gold standard method according to similar previous studies ${ }^{12-14}$, in order to visualize and describe all structures. Only three types of corpuscles were found, Golgi tendon organs, Pacinian-like corpuscles and free nerve endings. Unlike previous studies, we did not observe Ruffini's corpuscles or endings type I mechanoreceptors ${ }^{14}$. Golgi tendon organs were mainly observed near the distant thirds of the tendon, while samples from the middle part of the tendon appeared to contain mostly free nerve endings. Previous reports have described the majority of mechanoreceptors near the ends of the cruciate ligaments $2,7,13,19-21$, while others have shown that Golgi tendon organs are found in the middle of the tendon ${ }^{14}$

The findings mentioned above indicate that the mechanoreceptors do not appear by chance in various quantity and location across the tendon, but they seem to have a specific and significant role. The myotendinous and osteotendinous junctions are the parts of the tendon subjected to mechanical stress and involved in muscle movement and maintainance of the stability during the static orientation or during walking and running. We believe that these parts are more abundant in mechanoreceptors than the middle part, because their role is to transmit signals about direction of motion and position in space concerning such a crucial joint. The middle section of the tendon is not subjected to high pressure and this could explain the occurrence of free nerve endings whose function is the perception of pain and regulation of blood flow and it is ambiguous whether they should be considered as mechanoreceptors ${ }^{4,13}$. The results of our study are in agreement with the role of Golgi tendon organs that receive signals related to the direction of motion, position in space and importance of function. Also, it is important to mention the finding of Pacinian-like corpuscles which are presented quite different in appearance than the "usual" Pacinian corpuscles. Their existence is obvious if we consider that Pacinian corpuscles are generally responsible for sensitivity to vibration and pressure ${ }^{2}$. The majority and variance of mechanoreceptors in the distant thirds of the tendon may act as a self-protective trait which contributes to early response signals in various problems of the calcaneous and the muscles involved during the gait cycle.

The selection of these piglets was based on the correspondence of the anatomical and physiological characteristics of the animal with those observed in humans and thus, our study may have clinical application to the Achilles tendon of humans as well. It has been found that patients with a previous history of an Achilles tendon rupture display proprioception deficits $^{22}$. Furthermore, an impaired joint position sense at plantar flexion after endoscopically guided percutaneous Achilles tendon surgery has been proved $^{23}$. Interestingly, it has been shown that in Achilles tendon allografts there is no histological evidence of newly ingrown mechanoreceptors ${ }^{9}$. As regards to injured anterior cruciate ligament, it has been stated that its reconstruction restores proprioceptive potential of the knee to some extent and the remnant ligament contains residual mechanoreceptors, the number and functionality of these is dependent on the physical characteristics of the remnant and the duration of injury ${ }^{24}$.

Additionally a recent study showed that the plantar fascia, and its relationship with the paratenon of the Achilles tendon, has a role not only in supporting the longitudinal arch of the foot, but also in its proprioception and peripheral motor coordination ${ }^{25}$. All these should be taken into consideration before an Achilles tendon reconstruction is attempted and our findings may be the starting point for further research in humans.

In conclusion, this study showed that the Achilles tendon in pigs contains only three types of mechanoreceptors playing an important role in the normal function of the tendon. There have been described differ- 
ent neurosensory elements in different parts of the tendon, which indicate its functional role during any motion of the limb and not just a solid muoskeletal linkage. The findings presented above help us to understand and to investigate the role of the Achilles tendon in pigs. However, they can be used as a guide for the study of the proprioception of the human Achilles tendon. Further studies in animals and humans should be conducted in order to identify and describe all possible variations of mechanoreceptors and their relationship to the proprioception of the neuromuscular system.

\section{Acknowledgements}

There are no acknowledgments.

\section{Conflict of Interest}

The Authors declare that they have no conflict of interest.

\section{References}

1. Krause W. Die terminalen korperchen der eifach sensible Nerven. Anatomisch Physiologische Monographie. Hannover Hahn'sche Hofbuchlandlung. 1860.

2. Freeman MAR, Wyke B. The innervations of the knee joint. An anatomical and histological study in the cat. J Anat. 1967; 101:505-532.

3. Wada Y, Takohash T, Michinaka Y, Morisawa Y, Yamamoto $\mathrm{H}$. Mechanoreceptors of patellar tendon used for $\mathrm{ACL}$ reconstruction Acta orthop. Scandinavica. 1997;68(6):559562.

4. Hogervorst, et al. Mechanoreceptors in Joint Function. The $\mathrm{J}$ of bone and joint surgery American. 1998;80:1365-1378.

5. Bolanowski SJ, Zwislocki JJ. Intensity and frequency characteristics of Pacinian corpuscles. I. Action potentials. $J$ Neurophysiol. 1984;51:793-811.

6. Scott JJA. The Golgi tendon organ. In: Dyck PJ, Thomas PK editors. Peripheral Neuropathy. $4^{\text {th }}$ edn. Philadelphia: Elsevier-Saunders. 2005:151-161.

7. Banks RW. An allometric analysis of the number of muscle spindles in mammalian skeletal muscles. J Anat. 2006;208: 753-768.

8. Andres $\mathrm{KH}$, von During M, Schmidt RF. Sensory innervations of the Achilles tendon by group III and IV afferent fibers. Anat Embryol. 1985;172(2):145-156.

9. Kim SH, Chun CH, Chun KC, JO HJ, Kim KM. Histological assessment of mechanoreceptors in Achilles allografts anterior cruciate ligament reconstruction. Am J Sports Med. 2012;40(9):2061-2065
10. Nakamoto T, Matsukawa K. Muscle mechanosensitive receptors close to the myotendinus junction of the Achilles tendon elicit a pressor reflex. J Appl Physiol. 2007;102(6):21122120.

11. Padulo J, Oliva F, Frizziero A, Maffulli N. Muscles, Ligaments and Tendons Journal - Basic principles and recommendations in clinical and field science research: 2016 update. MLTJ. 2016;6(1):1-5.

12. O' Connor BL, Gonzales J. Mechanoreceptors of the medial collateral ligament of the cat knee joint. J Anat. 1979;129 (4):719-729.

13. Goertzen MJ, Clahsen H, Bürrig KF, Schulitz KP. Sterilisation of canine anterior cruciate allografts by gamma irradiation in argon. Mechanical and neurohistological properties retained one year after transplantation. J Bone Joint Surg Br. 1995:77(2):205-212.

14. Koch B, Kurriger G, Brand R.A. Characterisation of the neurosensory elements of the feline cranial cruciate ligament. J Anat. 1995; 187:353-359.

15. Morisawa Y. Morphological study of mechanoreceptors on the coracoacromial ligament. J orthop Science. 1998;3:102110.

16. Arcand MA, Rhalmi S, Rivard C-H. Quantification of mechanoreceptors in the canine anterior cruciate ligament. Orthopaedics. 2000;24:272-275.

17. Vangsness CT Jr, Ennis M, Taylor JG, Atkinson R. Neural anatomy of the glenohumeral ligaments, labrum, and subacromial bursa. Arthroscopy. 1995;11;180-184.

18. Zimny ML, Schutte M, Dabezies E. Mechanoreceptors in the human anterior cruciate ligament. Anat. Rec. 1986;14(2):204209.

19. Jazsa L, Balint J, Kannus P, et al. Mechanoreceptors in human myotendinous junction. Muscle Nerve. 1993;16:453457.

20. Kennedy JC, Weinberg HW, Wilson AS. The anatomy and function of the anterior cruciate ligament. J Bone Joint Surg. 1974;56A:223-244.

21. Schultz R, Miller D, Kerr C, Micheli I. Mechanoreceptors in human cruciate ligaments. J Bone Joint Surg [Am]. 1984 66:1072-1076.

22. Bressel E, Larsen BT, McNair PJ, Cronin J. Ankle joint proprioception and passive mechanical properties of the calf muscles after an Achilles tendon rupture: a comparison with matched controls. Clin Biomech (Bristol, Avon). 2004;19 (3):284-291.

23. Kaya D, Doral MN, Nyland J, Toprak U, Turhan E, Donmez G, Citaker S, Atay OA, Callaghan MJ. Proprioception leve after endoscopically guided percutaneous Achilles tendon. Knee Surg Sports Traumatol Arthrosc. 2013;21(6):12381244.

24. Dhillon MS, Bali K, Prabhakar S. Differences among mechanoreceptors in healthy and injured anterior cruciate ligaments and their clinical importance. Muscles Ligaments Tendons J. 2012 Jun 17;2(1):38-43.

25. Stecco C, Corradin M, Macchi V, Morra A, Porzionato A, Biz $C$, De Caro R. Plantar fascia anatomy and its relationship with Achilles tendon and paratenon. J Anat. 2013 Dec;223 (6):665-676 